\title{
Research Paper \\ Investigating the Effect of Economic Variables on Export of Iranian Traditional and Agricultural Goods
}

\author{
*Fariba Eghbalsefat Ronaghe ${ }^{1}$ (1), Nasibe Zare², Mohamadreza kohansal ${ }^{2}$
}

1. PhD. in Agricultural Economics, Department of Agricultural Economics, Faculty of Agricultural Engineering, Sari Agricultural Sciences and Natural Resources University, Sari, Iran.

2. Department of Agricultural Economics, Faculty of Agricultural, Ferdowsi University of Mashhad, Mashhad, Iran.

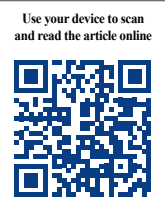

dititon: Eghbalsefat Ronaghe, F., Zare, N., kohansal, M. (2019). [Investigating the Effect of Economic Variables on Export of Iranian Traditional and Agricultural Goods (Persian)]. Quarterly Journal of the Macro and Strategic Policies, 7(2), 286-303. https://doi.org/10.32598/JMSP.7.2.286

oifhttps://doi.org/10.32598/JMSP.7.2.286

Received: 07 Oct 2017

Accepted: 06 Jan 2018

Available Online: 01 Jul 2019

Key words:

Exchange rate, Vector auto-regressive model, Money supply, Johansen method, Agricultural capital

\begin{abstract}
In this research, the effect of five macroeconomic variables of the exchange rate, gross domestic product, relative prices of agricultural commodities, agricultural capital, and money supply is investigated on the agricultural and traditional exports during 1986-2014. In the present study, we used the Vector Auto-Regressive (VAR) model and the Johansen method. The results show a positive and significant correlation between agricultural and traditional exports and variables of the exchange rate, relative prices, and money supply. However, there is a negative and significant relationship between agricultural capital and the dependent variable. Besides, there is a one-way causal relationship between the exchange rate and agricultural exports as well as the supply of money and agricultural exports. Finally, using the modified coefficient of Error-Correction Mechanism (ECM), we can infer that if an imbalance occurs in agricultural exports in any year, a quarter of imbalance will be restored in the next period. To determine the relationship between variables, in the long run, we used Johansson method to estimate the convergent vectors and apply constraints on cointegration relations. Based on the study results, we suggest that economic authorities should try to approximate the exchange rates and eliminate the gap between central bank exchange rate and free-market exchange rate. This method is the best way to stabilize the exchange rate, and consequently, export stability in the long run. To do this, the proper monetary and financial measures must be taken to direct liquidity toward production and boost the capital market.
\end{abstract}

\section{*Corresponding Author:}

\section{Fariba Eghbalsefat Ronaghe, $\mathrm{PhD}$.}

Address: Department of Agricultural Economics, Faculty of Agricultural Engineering, Sari Agricultural Sciences and Natural Resources University, Sari, Iran.

Tel: +98 (930) 0015185

E-mail: eghbalfariba@yahoo.com 


\title{
بررسى تأثير متغيرهاى اقتصادى بر صادرات كالاهاى سنتى و كثاورزى ايران
}

\author{
*فريبا اقبال صفت رونقى' (1)، نسيبه زارعى'، محمدرضا كهنسال' \\ 1 - دانشجوى دكتراى اقتصاد كشاوزى، كروه اقتصادكشاورزى، دانشكده كشاورزى، دانشعاه كشاورزى سارى، سارى، ايران

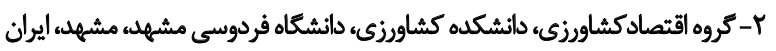

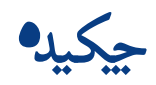

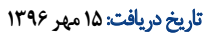

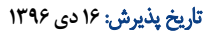

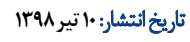

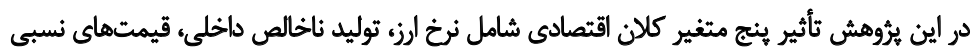

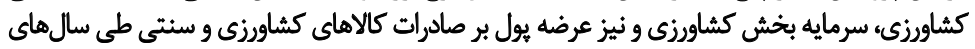

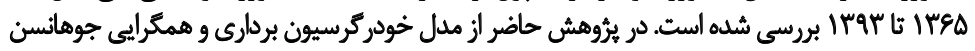

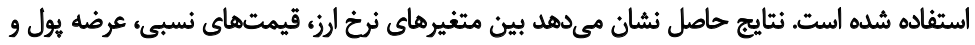

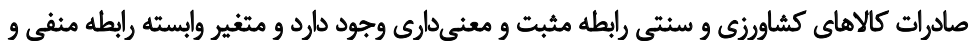

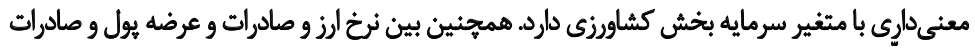

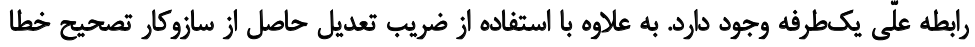

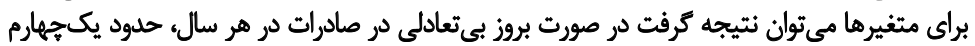

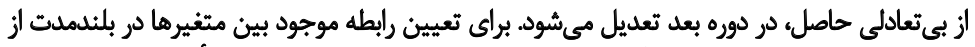

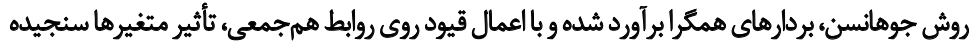

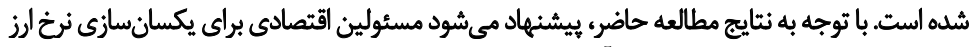

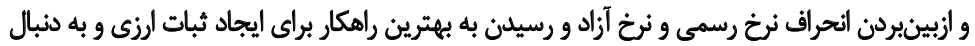

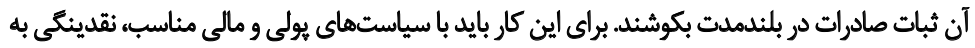

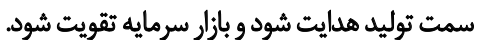

$:$ : $180 y$

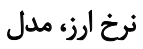

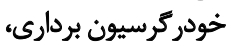

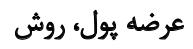

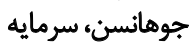
نحش كشاورزى
\end{abstract}




\section{dove}

در طول سالهاى اخير كوشش براى رسيدن به اهداف جشهمانداز · rالهاله و ايجاد ظرفيتهاى جديد براى

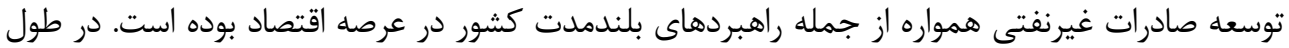

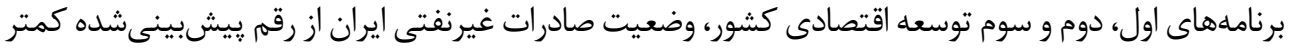

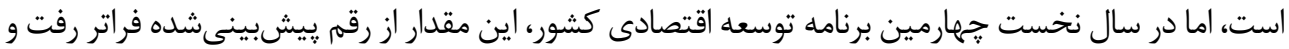

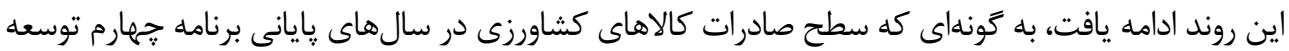

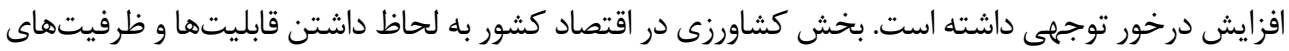

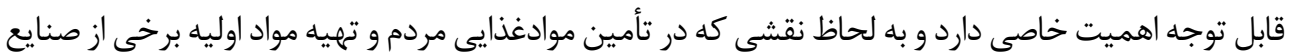
دارد، شايان توجه است.

در اين بين رسالت اصلى بخش كشاوزى به عنوان بخش توليدكننده غذا، تأمين نيازهاى غذايى افراد جامعه

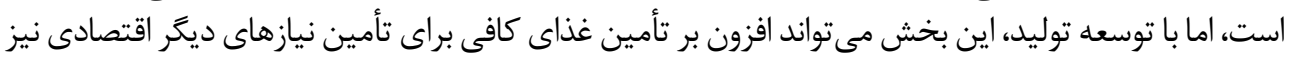

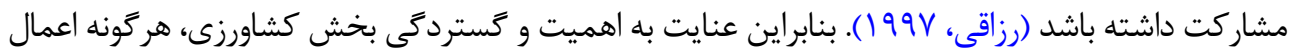

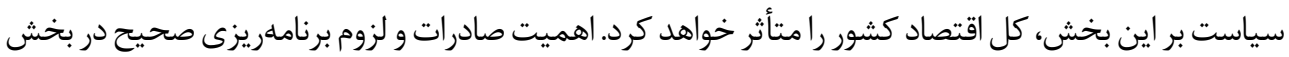

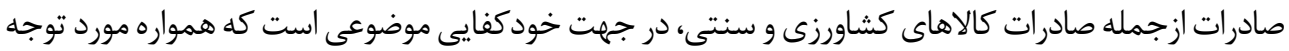

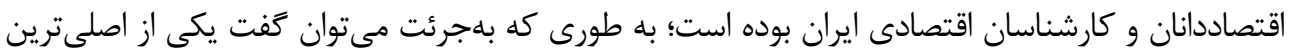

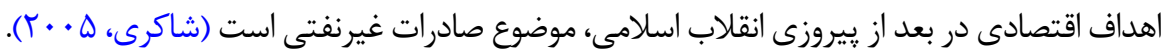
كسترش صادرات، عاملى است كه موجب سازماندهى توليد براى بازارهاى بزرى تر شده و درنتيجه روشهاى

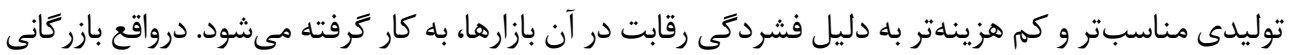

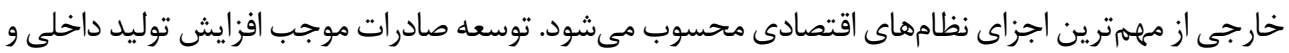

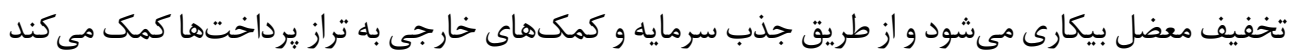

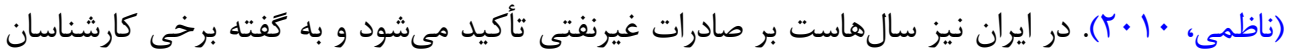

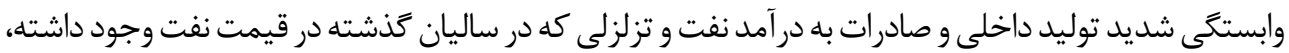

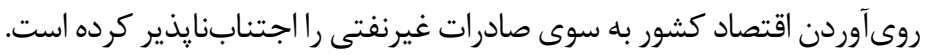

قابليتهاى فراوان كشور در زمينه توليد و عرضه محصولات كشاورزى سبب شده است اين محصولات حجم

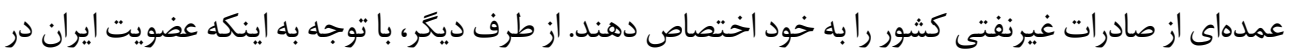

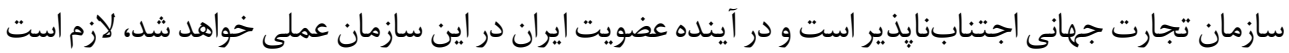

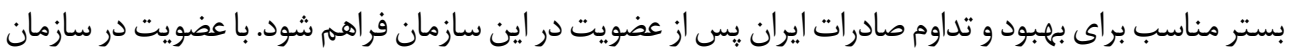

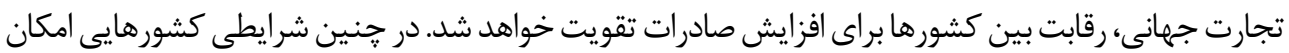

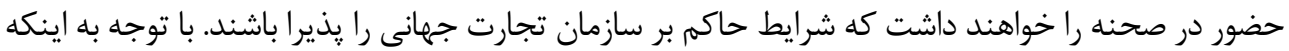

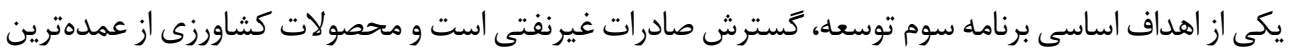

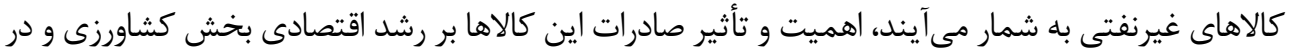


يى آن رشد اقتصادى كل كشور معين مىشود. هدف از يزوهش حاضر بررسى اثر متغيرهاى كلان اقتصادى بر

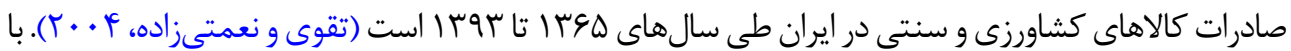

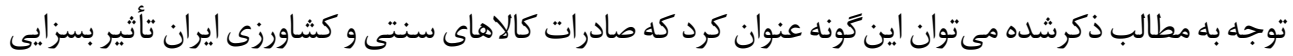

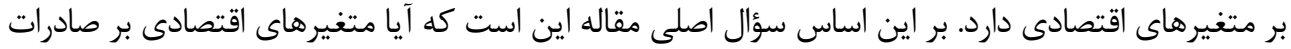

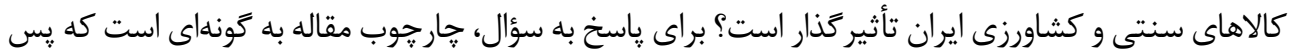

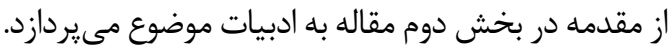

\section{1- ادييات موضوع}

در ارتباط بانرخ ارز، توليد ناخالص داخلى، قيمتهاى نسبى كشاورزى، سرمايه كشاورزى و نيز تأثير عرضه

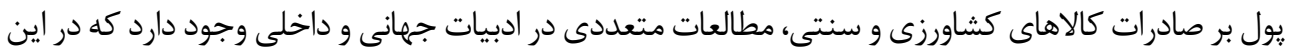

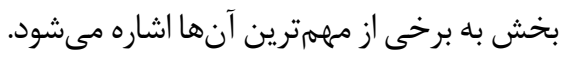

حسنوند و همكاران (19 • (ץ) تأثير تحريمها بر صادرات غيرنفتى ايران را با رويكرد سرى زمانى ساختارى، در

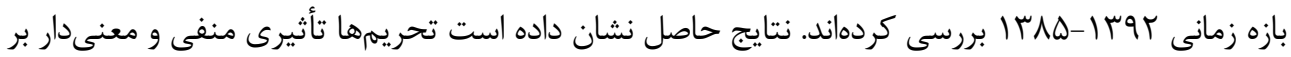

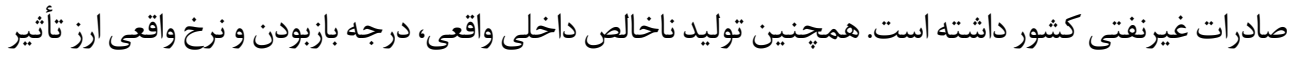

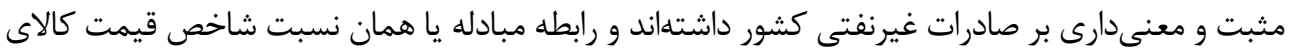

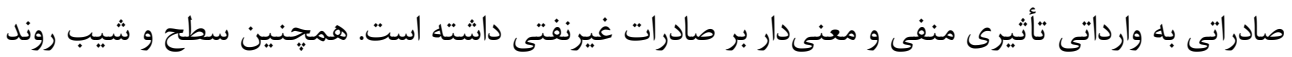

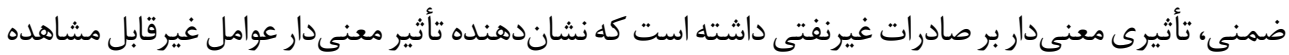

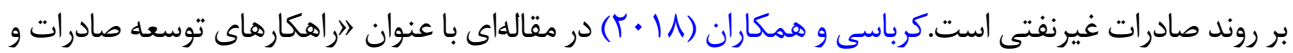

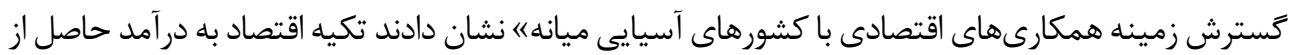
فروش نفت خام، بىثباتى درآمد صادراتى را به دنبال داردي فئه

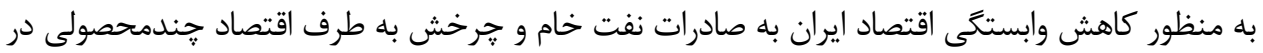

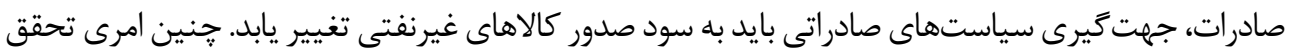

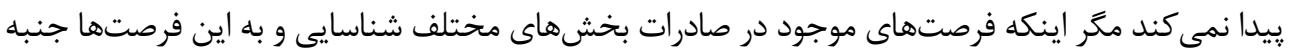

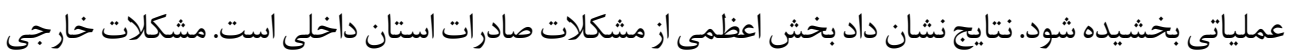

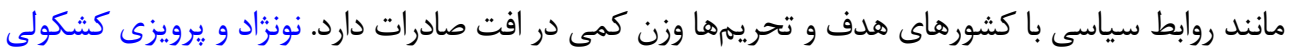

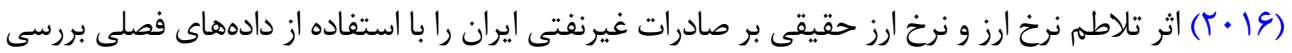

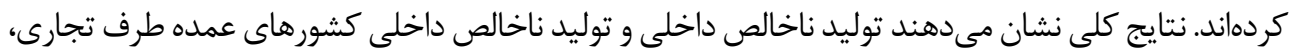

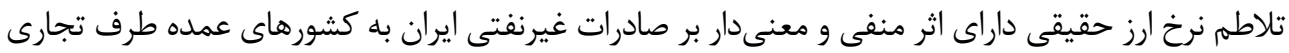

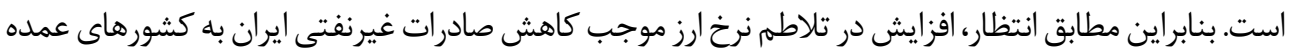

$$
\text { طرف تجارى مىشود. }
$$

محمدى و فكارى (ها • ץ) در مقالهاى با عنوان لاثرات زيرساختهاى نهادى و متغيرهاى كلان اقتصادى 


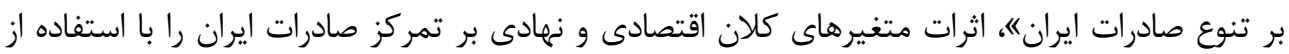

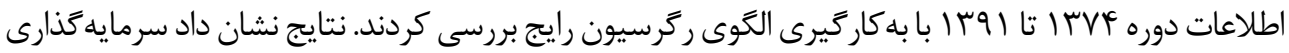

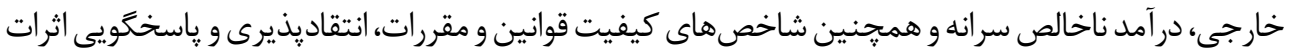

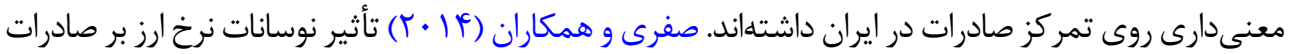

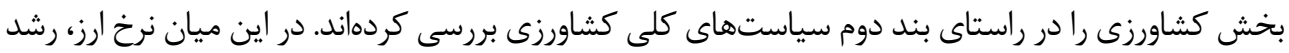

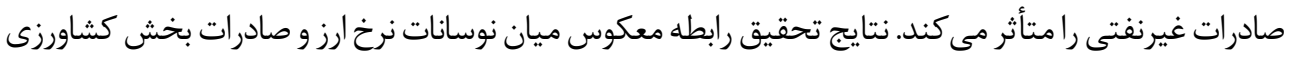
را نشان مىدهد.

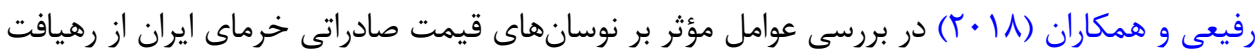

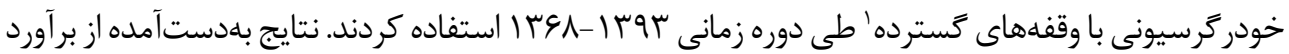

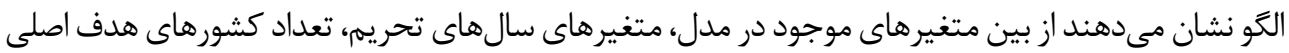

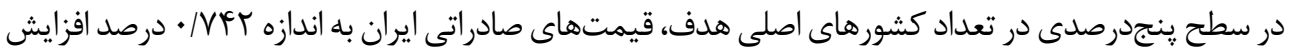

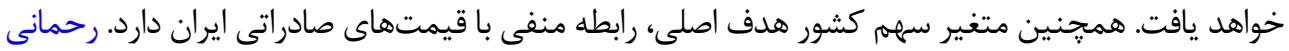

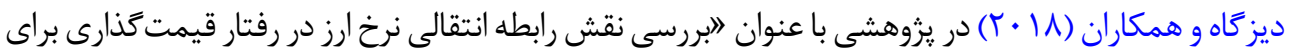

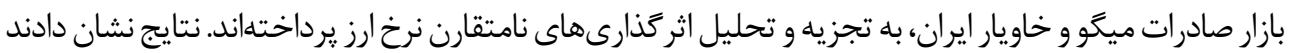

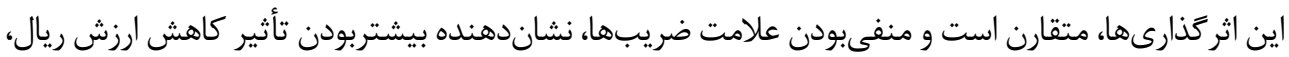

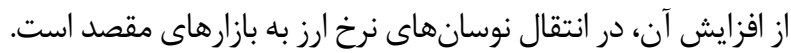

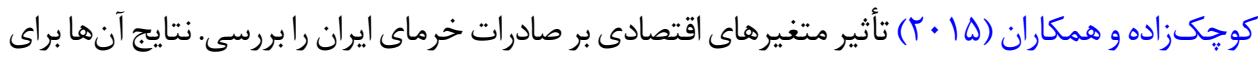

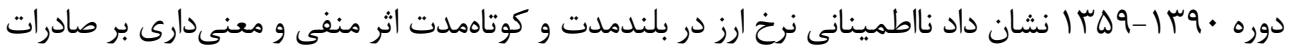

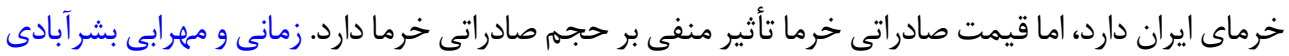

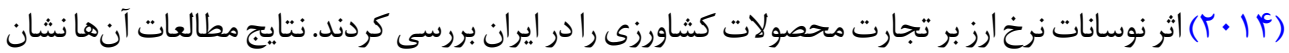

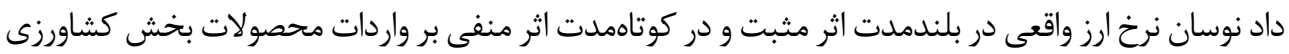

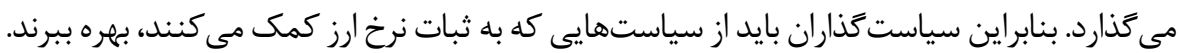

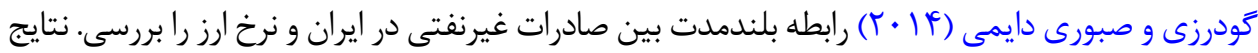

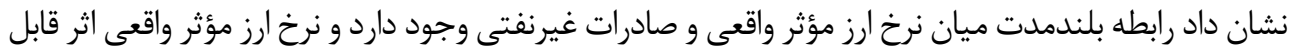

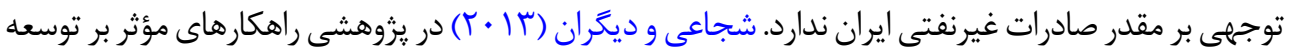

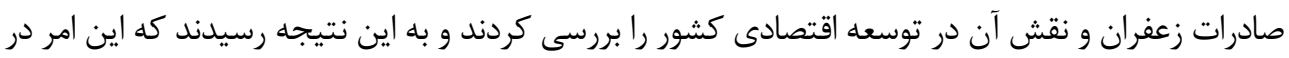

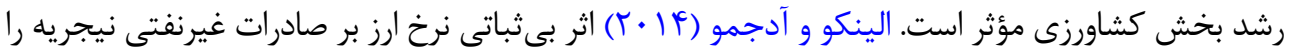

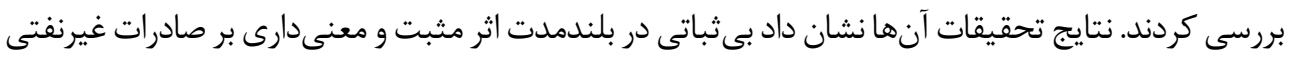
نيجريه دارد.

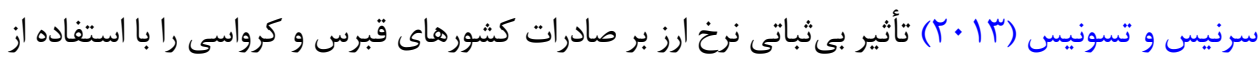

1. Autoregressive Distributed Lag (ARDL) 


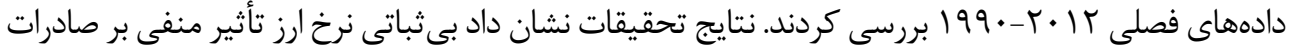

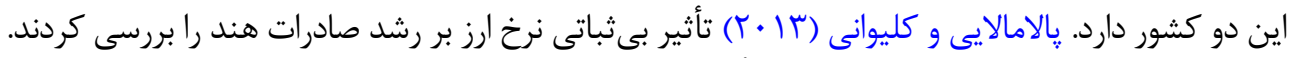

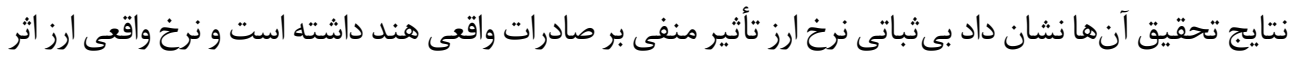

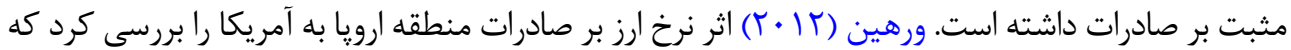

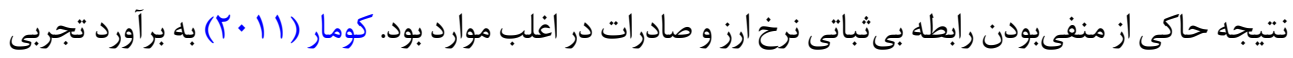

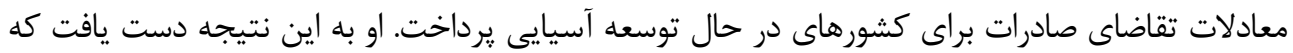

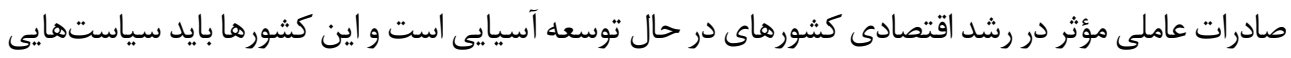

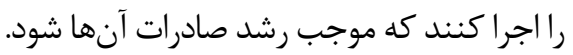

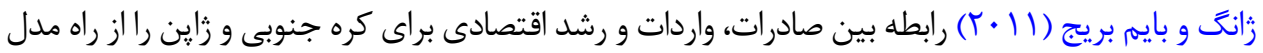

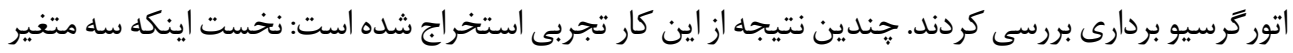

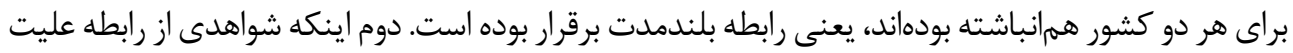

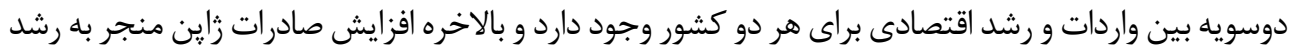

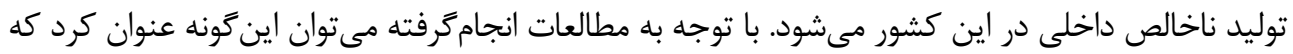

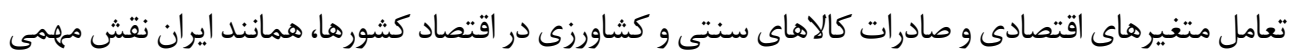

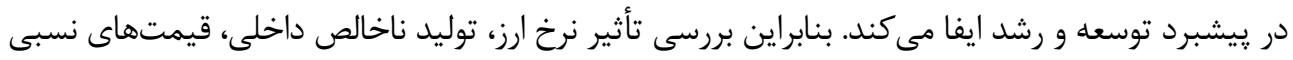

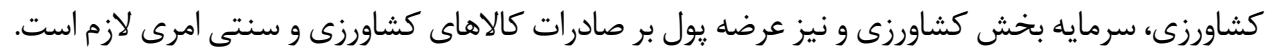

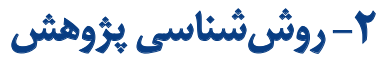

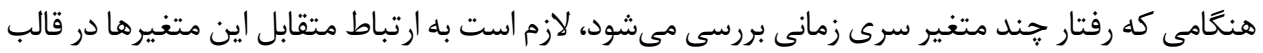

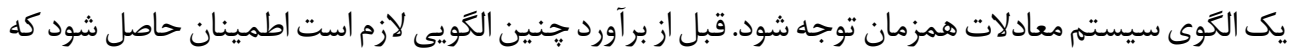

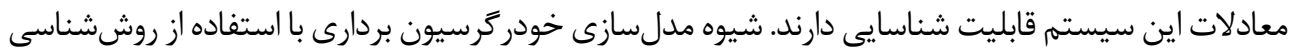

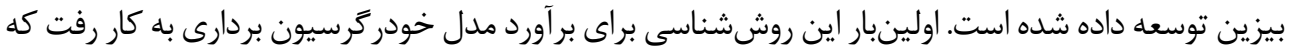

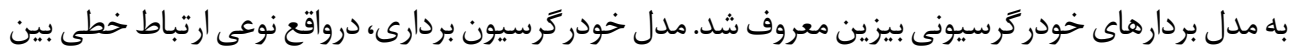

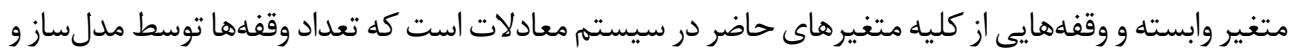

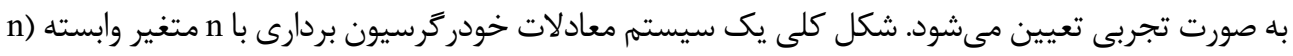
معادله) به شكل فرمول شماره است است:

\section{$Y t=A(L) Y t=C \varepsilon+t$}

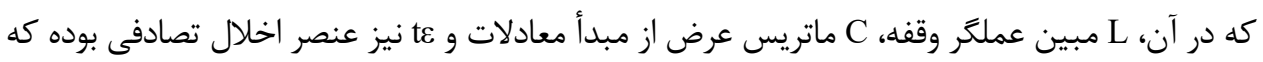

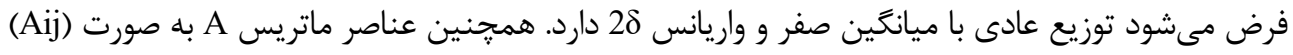
تعريف مىشوند (فرمول شماره ؟). 
$\operatorname{Aij}(L)=\sum L^{k} a_{i j k}$

كه در آن، أ معرف شماره معادله، j شماره متغير حاضر در معادله و k تعداد وقفه مدنظر براى سيستم است.

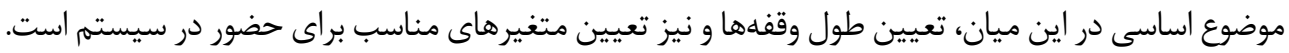

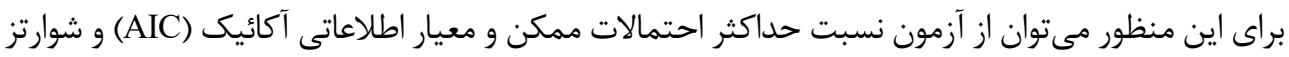

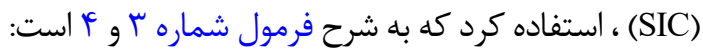

$A I C=T \log \left|\sum\right|+2 N$

$S I C=T \log \left|\sum\right|+N \log T$

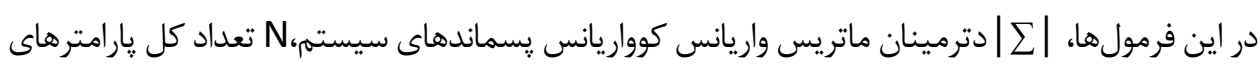

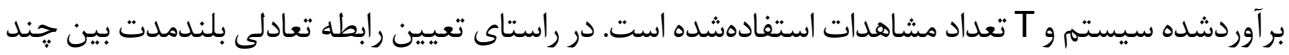

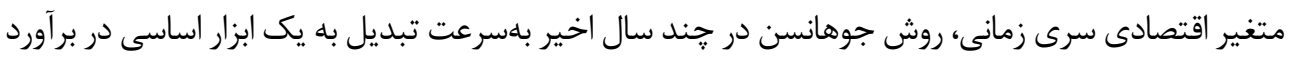

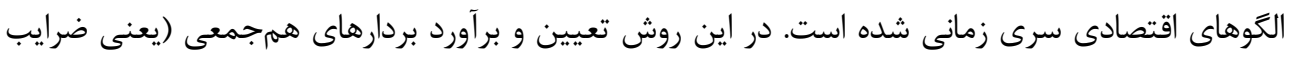

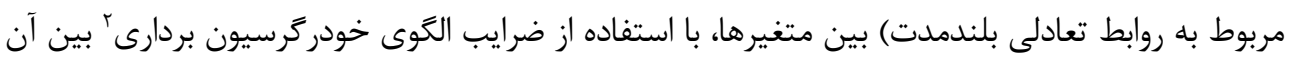

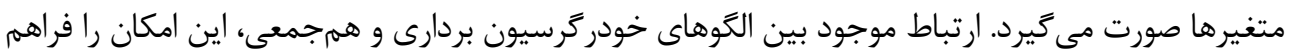

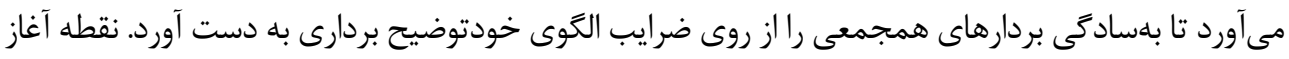

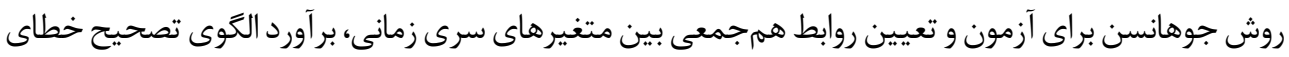

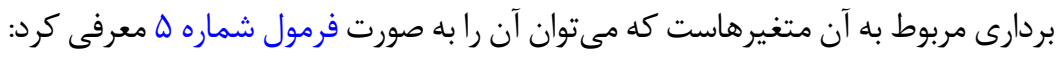

$$
\Delta y t=\beta_{1} \Delta y_{t-1}+\beta 2 \Delta_{t-2}+\ldots \ldots \ldots . .+\beta_{p-1} \Delta y_{t-p-1}+I I y_{t-p}+U_{t}
$$

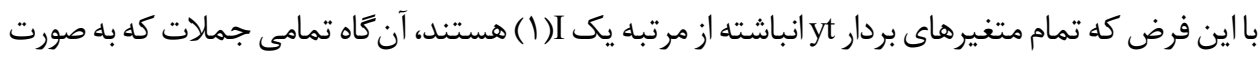

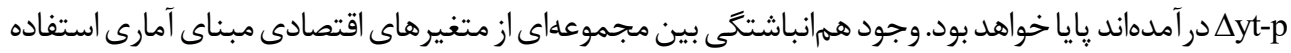

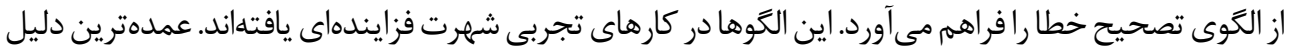

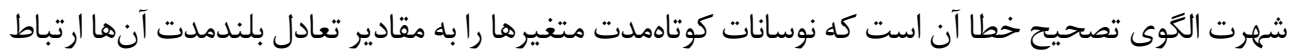

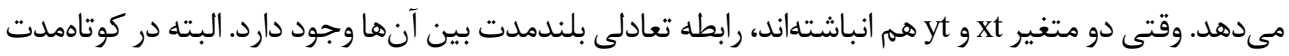
ممكن است بى تعادلىهايى وجود داشته باشد، در اين صورت مىتوان جمله اخلال فرمول شماره و و V را بانه 


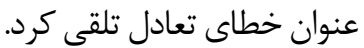

$Y t=\beta x_{t}+U_{t}$

$U t=y_{t}-\beta x_{t}$

اينكى مى توان اين خطا را براى ييوند رفتار كوتامدت لyt با مقدار تعادلى بلندمدت آن استفاده كرد و براى اين

منظور مىتوان الكويى به صورت فرمول شماره 1 تنظيم كرد:

$$
\Delta y_{t}=\alpha_{0}+\alpha_{1} \Delta x_{t}+\alpha_{2} \hat{U}_{t-1}+\varepsilon_{t}
$$

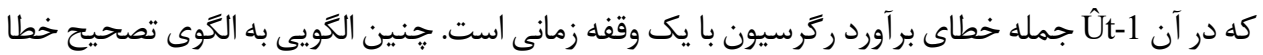

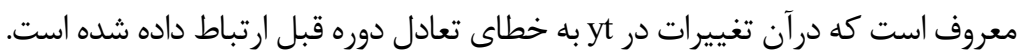

براى تخمين مدل مطالعهشده، يعنى تابع صادرات كالاهاى سنتى و كشاورزى، از فرمول شماره 9 استفاده

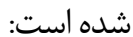

$$
X_{t}=f\left(E R_{t} P W_{t} y_{i} I N_{t}, M\right)
$$

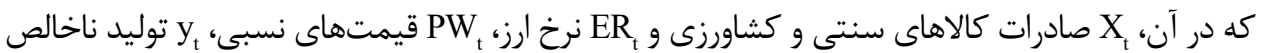

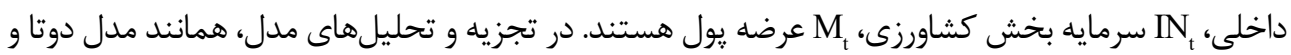

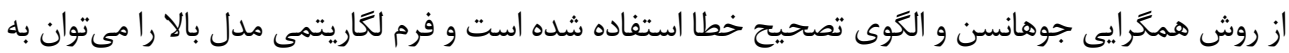
صورت فرمول شماره • إنشان داد:

$\operatorname{Ln} X_{t}=f\left(\operatorname{Ln} E R_{t} \operatorname{Ln} P W_{t}\right.$ Ln $\left.y_{i} \operatorname{Ln} I N_{t} \operatorname{Ln} M_{t}\right)$ كه اين رابطه را مىتوان به صورت فرمول شماره 11 براى برآورد مدل بازسازى كرد:

$\operatorname{Ln} X_{t}=\alpha_{0}+\alpha_{1} \operatorname{Ln} E R_{t}+\alpha_{2} \operatorname{Ln} P W_{t}+\alpha 3 \operatorname{Ln} y_{t}+\alpha_{4} \operatorname{Ln} I N_{t}+\alpha_{5} \operatorname{Ln} M_{t}$

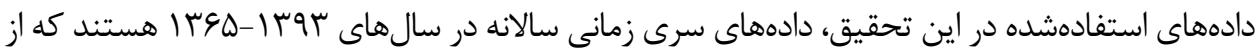


جدول 1. آزمون ريشه واحد براى متغيرهاى تحقيق با استفاده از روش ديكىفولر

\begin{tabular}{|c|c|c|c|c|c|}
\hline \multirow{2}{*}{$\begin{array}{l}\text { تثيجه آزمون } \\
\text { ADF }\end{array}$} & \multicolumn{3}{|c|}{ مقدار بحراني ADF (درصد) } & \multirow{2}{*}{ آماره آزمون ADF } & \multirow{2}{*}{ 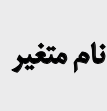 } \\
\hline & 1 & - & 1. & & \\
\hline$I(1)$ & $-r / N E q$ & $-r /+*$ & -t/ATt & $-r / f i r$ & $\mathbf{L X}$ \\
\hline$I(1)$ & $-r / V M$ & $-4 /+11$ & -r/gFe & $-4 / .90$ & LER \\
\hline$I(1)$ & $-1 / \Delta A r$ & $-T / M T$. & $-r\left(\lambda_{*}\right)$ & $-r / A r Q$ & LPW \\
\hline$I(1)$ & $-r / \Lambda \Delta V$ & $-r /+r$ & $-r / 98$ & -r/ArA & LY \\
\hline$I(1)$ & $-H / A \Delta V$ & $-r / * \rho_{0}$ & -T/88. & $-\$ 1119$ & LIN \\
\hline$I(1)$ & $-T / N E q$ & $-r / \cdot$. & - rlaper & -T/AFq & LM \\
\hline
\end{tabular}

(4)

مأخذ: محاسبات تحقيق

بانك مركزى ايران جمعآورى شده است. اين مقاله در سال هوسا نوشته شده است. همجنين آمارهاى ارائهشده در ارتباط با مطالعه حاضر از سوى

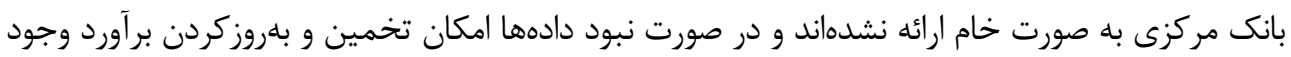
نخواهد داشت.

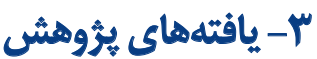

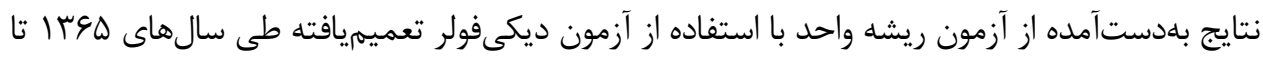

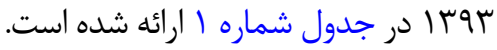

بر اساس جدول شماره ا تمامى متغيرها با اعمال يك وقفه يايا مىشوند و همگى انباشته از مرتبه يك ا(1)

جدول r r تعيين تعداد وقفه بهينه براي متغيرها، مدل خودركرسيون بردارى

\begin{tabular}{|c|c|c|c|}
\hline حثان كوئين & آكائيك & شوارتز بيزين & طول وقفه \\
\hline I/Pr & $1 / F a A$ & $V / \Delta \cdot V$ & . \\
\hline -. & $-+/$ TFA & $-. / 11<9$ & 1 \\
\hline . /FAF & 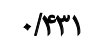 &.$- / \Delta \Psi$. & $r$ \\
\hline.$/ 9 \Delta$ &.$/ 9 Y A$ & $V \cdot r A$ & $r$ \\
\hline
\end{tabular}

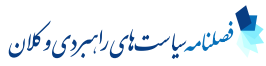

مأخذ: محاسبات تحقيق 
Trace $\lambda$ جدول r. آزمون ماتريس اثر

\begin{tabular}{|c|c|c|c|c|}
\hline الرزش احتمالي در سطح هو & مقدار بحراتي در سطح معنادارى & مقدار آماره آزمون & فرضيه مقابل & فرضيه صفر \\
\hline $.1 .$. & PVIASE & $N \Delta / T M$ & $r \geq 1$ & $r=$ \\
\hline .1 .08 & ra/var & MVI.TD & $r \geq r$ & $r \leq 1$ \\
\hline . IrTq & $10 / \& q f$ & $1 . M M$ & $r \geq r$ & $r S r$ \\
\hline.$/ 94$. & rafi & $.1 \cdots$ & $r \geq r$ & $r \leq r$ \\
\hline
\end{tabular}

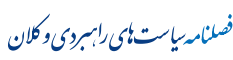

مأحذ: هحاسبات تحقيق هستند؛ جراكه در تفاضل مرتبه اول لعاريتمى تمامى متغيرها، مقدار كميت آمارى از لحاظ قدر مطلق بزرگتر

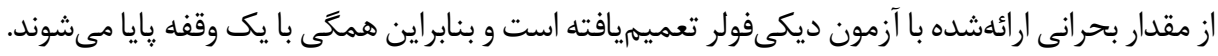
حالا بايد مرتبه بهينه مدل خودر گرسيون بردارى با استفاده از ملاكهاى تعيين وقفه معين شود. تعيين وقفه

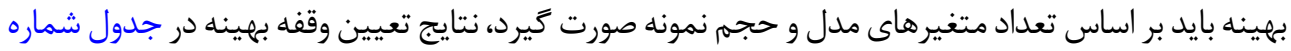
r ارائه شده است باست بر اساست

با توجه به جدول شماره ז، آماره شوارتز بيزين، آكائيك و حنان كوئين در وقفه يك، كمترين مقدار را

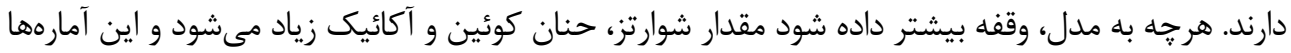

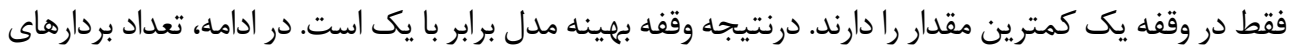

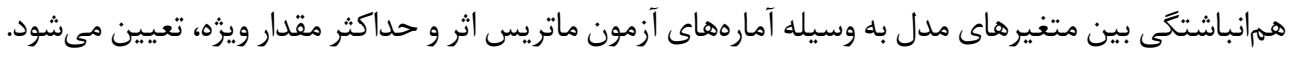

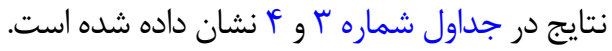

با توجه به نتايج جداول شماره ؟ و أ، وجود دو رابطه بلندمدت ميان متغيرهاى مدل نشان داده شده است.

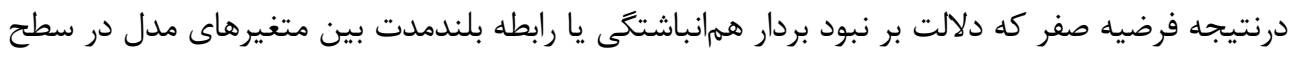

Max $\lambda$

\begin{tabular}{|c|c|c|c|c|}
\hline ارزش احتمالى در سطح & مقدار بحرانى در سطح معنادارى & مقدار آماره آزمون & فرضيه مقابل & فرضيه صفر \\
\hline$\%$ & TV/AAF & FNMTV & $r=1$ & $r=$ \\
\hline$\cdot 1 \cdot+1$ & YM/IMI & TE/MTH & $r=r$ & $r \leq 1$ \\
\hline .1189 & If/refe & $1 \cdot N \cdot \Delta$ & $r=r$ & $r \leq r$ \\
\hline.$/ 94$. & $r / A F^{e}$ & $.1 . .0$ & $r=r^{e}$ & $r \leq r$ \\
\hline
\end{tabular}

|ct

مأخذ: هحاسبات تحقيق 
جدول هـ نتايج استخراج آزمون تصحيح خطا

\begin{tabular}{|c|c|c|c|}
\hline 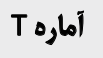 & انحراف معيار & ضريب & متغير \\
\hline$r / A N^{n}$ &.$/ M E D$ &.$- / 919 q$ & $\mathrm{D}(\mathrm{LX}(-1))$ \\
\hline r/ATr & I/TAF & $\Delta / 1 V \bar{Q}$ & D(LER(-1)) \\
\hline.$- / 119$ & . AATY & -.1190 & $\mathrm{D}(\operatorname{LPW}(-1))$ \\
\hline I/FAD & . & - $1 \cdot \Delta T$ & $\mathrm{D}(\mathrm{LY}(-1))$ \\
\hline . NaP & .1 .811 &.$/ . \Delta p q$ & $\mathrm{D}(\operatorname{LIN}(-1))$ \\
\hline $.1 \cdot 48$ &. $\mid 101$ & $+1 \cdot \cdot V$ & $\mathrm{D}(\mathrm{LM}(-1))$ \\
\hline- /ADF & +lapq & $-+/ R T$ & $\mathrm{C}$ \\
\hline - r/Ar &.$/ 94$ & $-+/ T E$ & ECM(-1) \\
\hline
\end{tabular}

معنى دارى ينج درصد است رد مى شود و فرضيه مقابل كه بر وجود رابطه بلندمدت بين متغيرهاى مدل است،

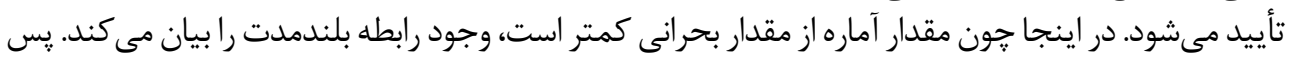

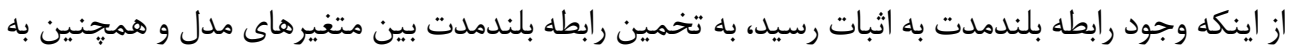

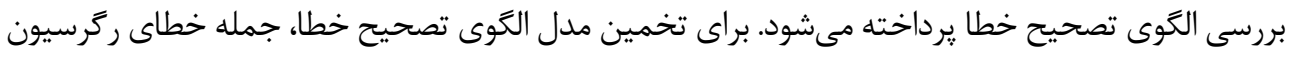

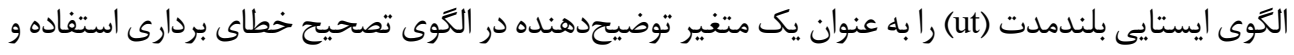

جدول و. استخراج رابطه بلندمدت بين مدل

\begin{tabular}{|c|c|c|c|}
\hline T آهاره T & انحر أف معيار & ضريب & متغيرها \\
\hline- & - & -1 & LX \\
\hline$r / \Delta \Delta A$ &.$/ 1 \triangle A$ & $+/ 4+\Delta$ & LER \\
\hline$-r / r+R$ & $.1 . e r$ & -./1ire & LPW \\
\hline$V / \perp \Delta \Delta$ &.$/ 1 T q$ & . /9re & LY \\
\hline r/Tre &.$/ 110$ & +pera & LIN \\
\hline r/\&AV & THT & - /AAQ & LM \\
\hline$-r / M$ & NMTr & -rT/Tqs & $\mathrm{C}$ \\
\hline
\end{tabular}

|ct

مأخذ: محاسبات تحقيق 
ضريب جمله تصحيح خطا نشان مى دهد، در هر دوره جند درصد از بى تعادلى متغير وابسته به سمت رابطه

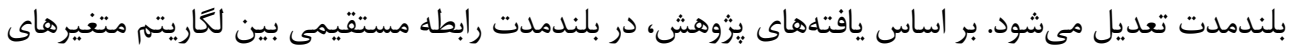

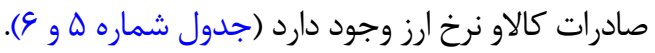

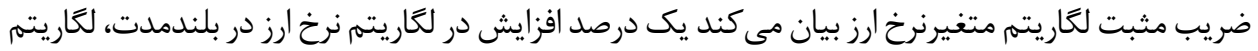

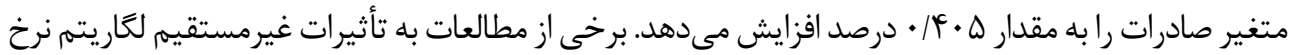

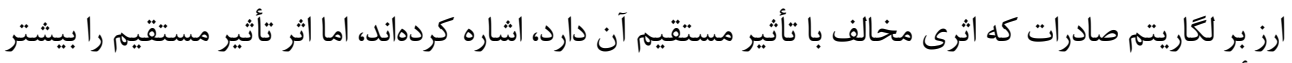

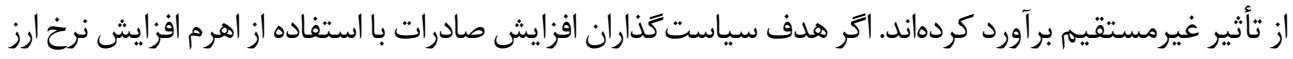

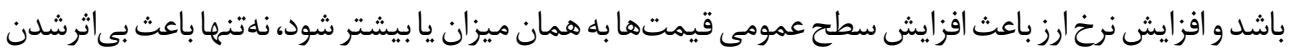

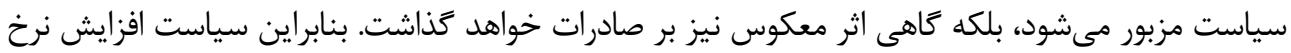

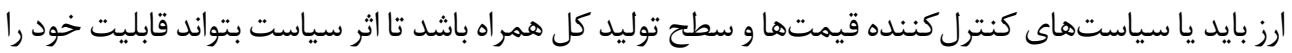
نشان دهد.

راهحل خروج از افت صادرات را بسيارى از كارشناسان و مديران كسبوكار، افزايش نرخ ارز ميىدانند؛ زيرا

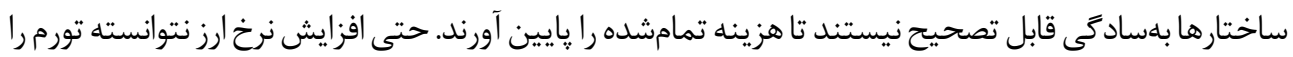

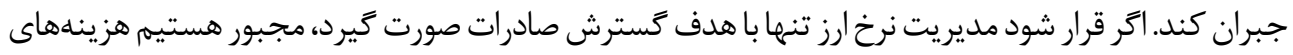

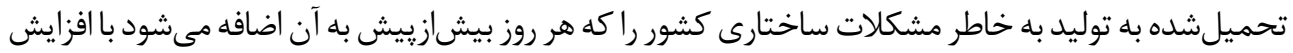

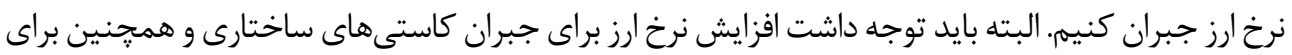
جبران تورم موجب افزايش قيمت واردات مىشود كه خود باعث افزايش قيمت تمامشده كالا و خدمات توليدى إنى

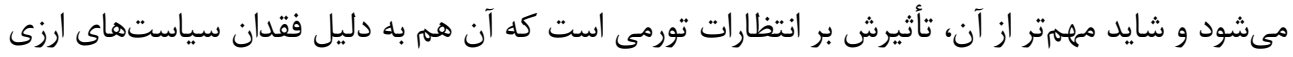

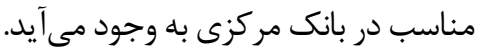

همجنين رابطه غيرمستقيمى بين قيمتهاى نسبى و صادرات كالاو جود دارد. ضريب منفى قيمتهاى نسبى

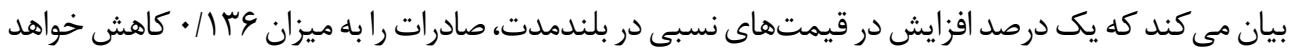

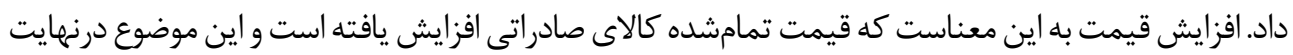

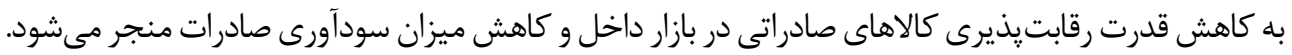

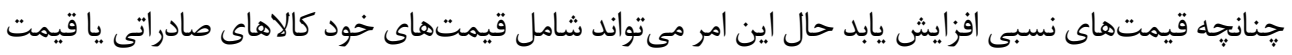

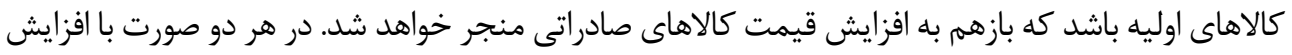

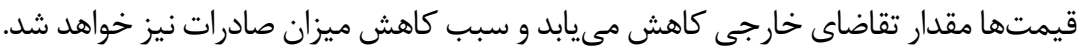

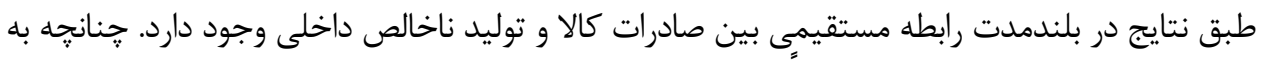

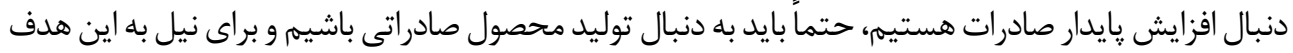

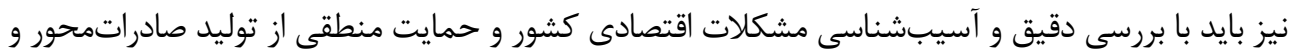

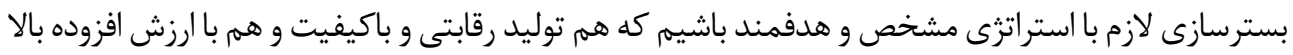




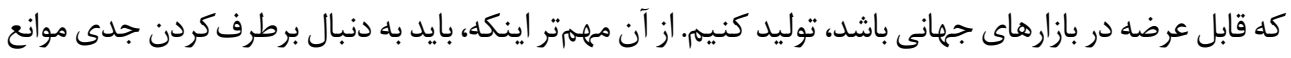

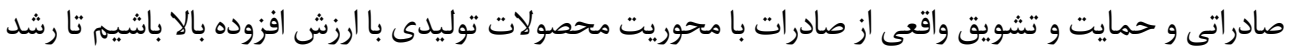
واقعى صادرات نسبت به واردات ران ادر عمل مشاهده كنيتم.

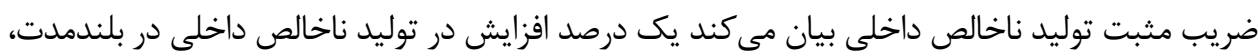

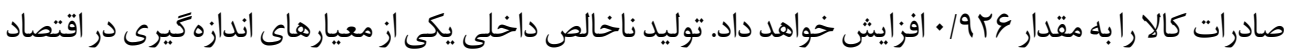

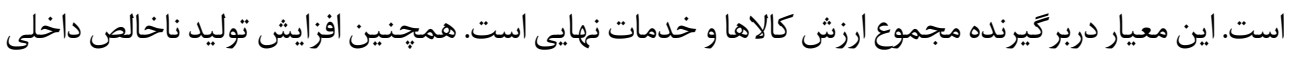

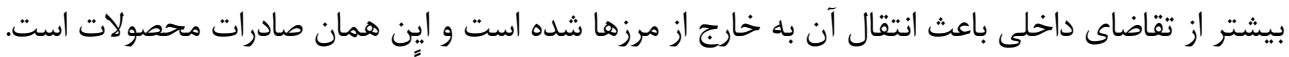

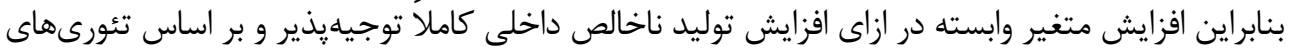

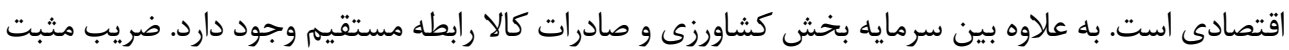

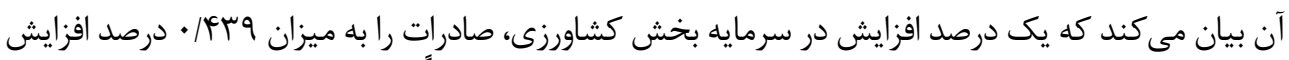

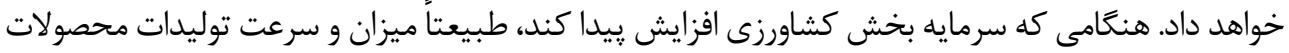

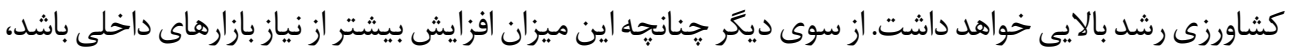

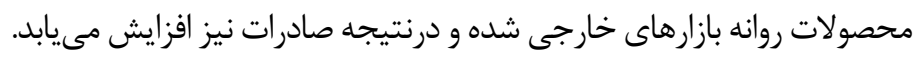

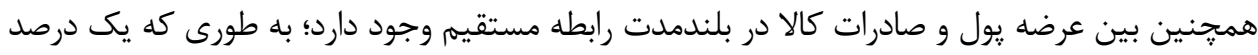

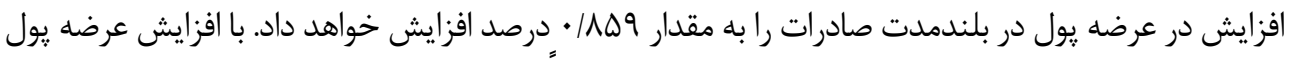

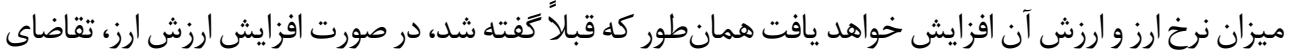

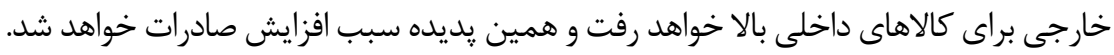

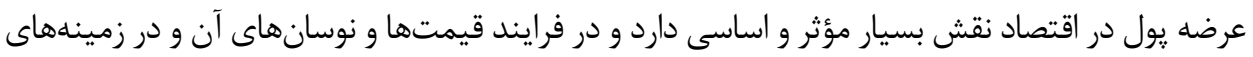

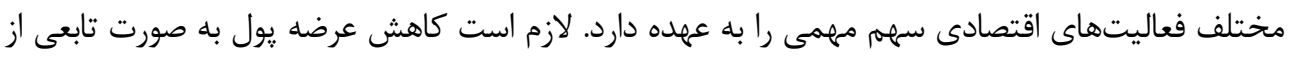

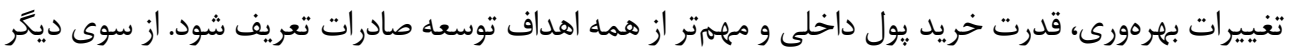

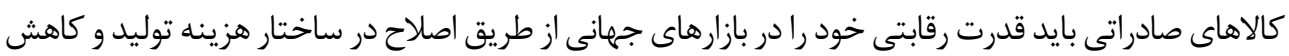

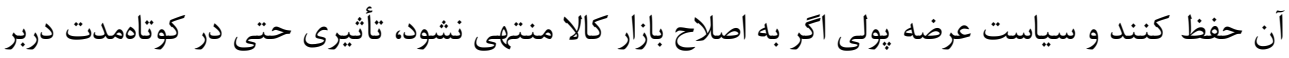
نخواهد داشت.

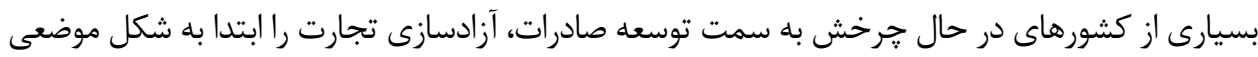

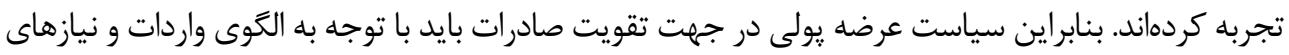

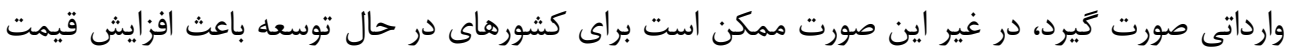

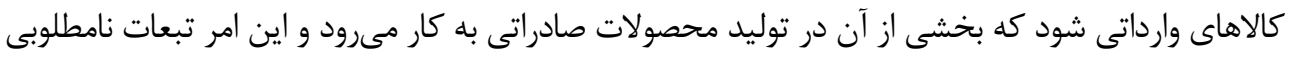

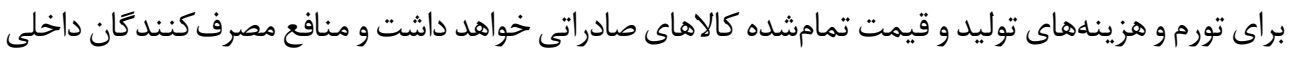

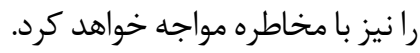




\section{ع - بحث ونتيجليَيرى}

در مقاله حاضر، تأثير متغيرهاى اقتصادى بر صادرات كالاهاى سنتى و كشاورزى ايران تجزيه و تحليل شده

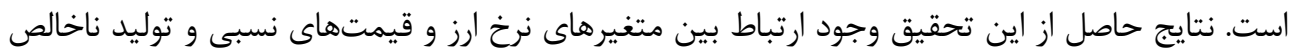

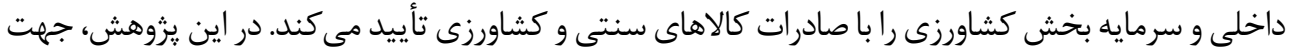

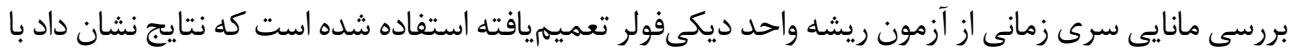

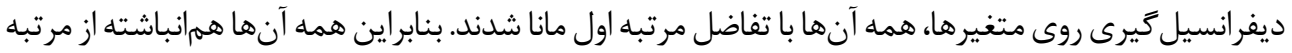

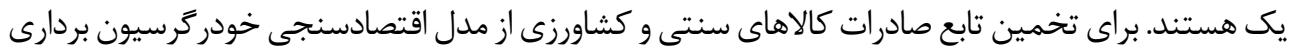

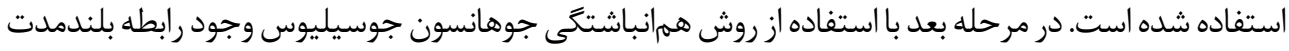

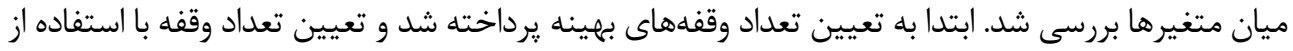

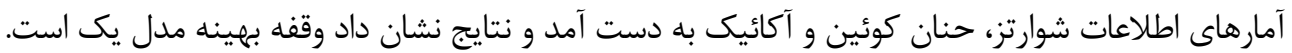

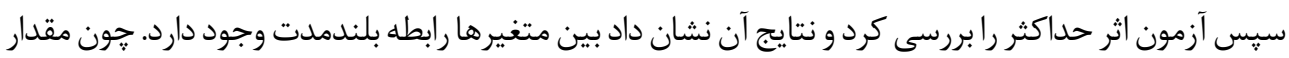

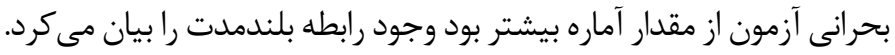
تابع صادرات تخمينزدهشده متغيرهاى معنى دار مقدار سرمايه، ميزان توليد ناخالص داخلى، شاخص قيمت

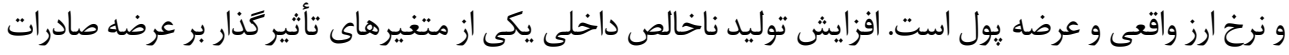

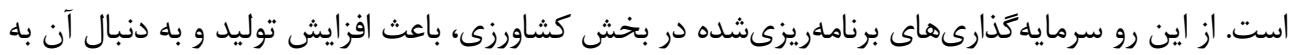

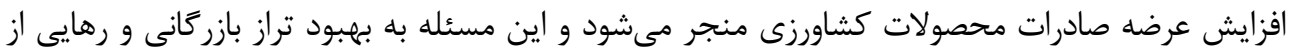

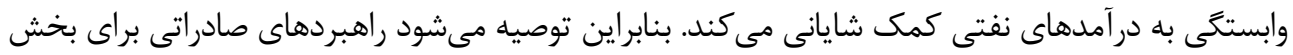

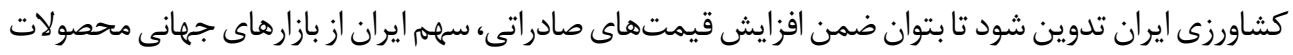

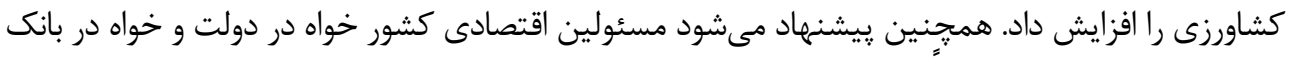

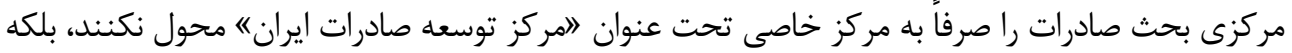

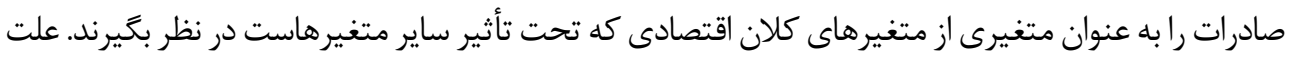

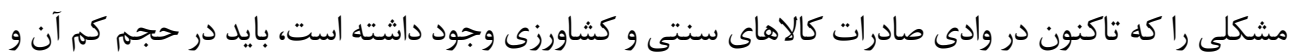

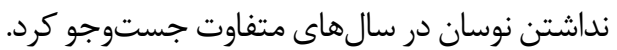

مالاحظاث اخلاقي

\section{ييروى از اصول اخلاق يُؤهش}

همه اصول اخلاقى در اين مقاله رعايت شده است. شركت كنند

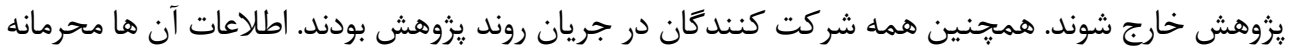




$$
\text { ماهي مالي ماين مقاله حامى مالى ندارد. }
$$




\section{References}

Akinlo, A. E., \& Adejumo, V. A. (2014). Exchange rate volatility and non-oil exports in Nigeria: 1986-2008. International Business and Management, 9(2), 70-9. [DOI:10.3968/5726]

Goodarzi, A., \& Sabouri Deylami, M. H. (2014). [Investigating the Long-term relationship between exchange rates and non-oil exports in Iran (Persian)]. Majlis \& Rahbord, 21(77), 39-5.

Hassanvand, A. A., Hassanvand, D., \& Nademi, Y. (2019). [The Impact of sanctions on non-oil exports of Iran: Structural time series approach (Persian)]. Quarterly Journal of The Macro and Strategic Policies, 6(24), 121-40.

Karbasi, A. R., Mohammadzadeh, S. H., Rasoolian, A., \& Ashrafi, M. (2018). [Export development strategies and expanding economic cooperation with central asian countries (Persian)]. Quarterly Journal of The Macro and Strategic Policies, 6(21), 100-21.

Koochakzadeh, A., Jalaei Esfand Abadi, A., \& Koochakzade, S. (2015). [The effects of exchange rate uncertainty on export of Iranian date (Persian)]. Journal of Agricultural Economics Research, 7(25), 157-71.

Kumar, S. (2011). Estimating export demand equations in selected Asian countries. Journal of Chinese Economic and Foreign Trade Studies, 4(1), 5-16. [DOI:10.1108/17544401111106770]

Mohammadi, H., \& Fakari, B. (2015). [Analyzing the effect of the institutional infrastructure and macroeconomic variables on the diversity of Iran's exports using ridge regression (Persian)]. Quarterly Journal of The Macro and Strategic Policies, 3(11), 75-94.

Nazemi, F. (2010). [Effect of macroeconomic variables on non-oil exports. Industrial Management Journal of Islamic Azad University (Persian)]. Journal of Industrial Management Faculty of Humanities Islamic Azad University, Sanandaj Branch, 4 (10), 105-17.

Nonejad, M., \& Parvizi Kashkouli, F. (2016). [The effect of exchange rate volatility on the none-oil exports of iran to major trade partners (Persian)]. Quarterly Journal of The Macro and Strategic Policies, 3(12), 99-122.

Palamalai, S., \& Kalaivani, M. (2013). Exchange rate volatility and export growth in India: An ARDL bounds testing approach. Decision Science Letters, 2(3), 191-202. [DOI:10.5267/j.ds1.2013.04.002]

Rafie, H., Mirbagheri, Sh., Akbarpour, H. (2018). [Investigating factors affecting export price volatility of Iran's date (Persian)]. Journal of Agricultural Economics Research, 10(37), 149-70.

Rahmani Dizghah, M., Mortazavi, S. A., Mosaviorcid, H. A. (2018). [The role of exchange rate pass-through at pricing to market behavior of iran's major export shrimp and caviar (Persian)]. Agricultural Economics, 11(4), 107-25.

Razaghi, A. (1997). [Introduction to Iranian economy (Persian)]. Tehran: Ney.

Safari, S., Rahmani, M., Ahmadi, H. (2014). [Effect of exchange rate volatility on agricultural exports (according to agricultural general policies) (Persian)]. Quarterly Journal of The Macro and Strategic Policies, 2(5), 97-109.

Serenis, D., \& Tsounis, N. (2013). Exchange rate volatility and foreign trade: The case for Cyprus and Croatia. Procedia Economics and Finance, 5, 677-85. [DOI:10.1016/S2212-5671(13)00079-8]

Shakeri, A. (2005). [Non-oil exports determinants (Persian)]. Iranian Journal of Economic Research, 6(21), 23-50.

Shojaei, B., \& Rasouli, A., Anowie Takie, L. (2013). [Study of effective strategies for export development of saffron and its role in economic development of the country (Persian)]. Papre presented at 1st National Conference on Medicinal Plants and Sustainable Agriculture, Hamadan, Iran, 10 October 2013. 
Taghavi, M., \& Nemati Zadeh, S. (2004). [The effect of macroeconomic variables on non-oil exports in Iranian economy (Persian)]. Journal of Economic Research, 4(14), 71-95.

Verheyen, F. (2012). Bilateral exports from euro zone countries to the US - Does exchange rate variability play a role? International Review of Economics \& Finance, 24, 97-108. [DOI:10.1016/j.iref.2012.01.007]

Zamani, F., \& Mehrabi Basharabadi, h. (2014). [The effects of exchange rate volatility on agricultural trade in Iran (Persian)]. Journal of Agricultural Economics Research, 6(22), 13-28.

Zang, W., \& Bainbridge, M. (2011). Exports, imports and economic growth in South Korea and Japan: A tale of two economics. Applied Economics, 44(3), 361-72. [DOI:10.1080/00036846.2010.508722] 
This Page Intentionally Left Blank 